\title{
Alternative Solutions to Kinetic Stimulus Transformations
}

\author{
Irvin Rock and Deborah Smith \\ Institute for Cognitive Studies, Rutgers-The State University
}

\begin{abstract}
It has been assumed that certain stimulus transformations lead directly to depth effects, that is, that such transformations are the necessary and sufficient conditions for kinetically generated depth perception. An alternative is to view such perception as the preferred solution to the problem posed by the transforming stimulus as to what event in the world is producing that transformation. In several experiments it is shown that when other solutions are supportable by the stimulus, those same transformations will no longer lead to depth perception. These other solutions become preferred on the basis of rejection of certain coincidental features of the stimulus that otherwise would have to be accepted were the kinetic depth solution to be maintained. The findings are interpreted as challenging any theory that perception is simply the direct result of stimulation or of extraction of stimulus information and as supporting the Helmholtzian rule of perception as a construction of the most reasonable representation.
\end{abstract}

Whenever one moves about in the environment, the retinal images of objects undergo continuous transformation. In the laboratory situation, with the observer stationary, Wallach and O'Connell (1953) have simulated these changing patterns of retinal stimulation. They rotated thin wire objects behind a screen, and observers viewed the transforming shadow patterns cast by such objects. The subjects typically perceived three-dimensional objects rather than the logically possible alternative of distorting two-dimensional configurations. Johansson and Jansson (1968) have achieved similar effects using an animated film technique. This phenomenon is referred to as the kinetic depth effect. Wallach and O'Connell believed that the effect depends on the simultaneous change of length and orientation of the figure's retinal projection. Although the presence of orientation change simultaneous with length change is not absolutely necessary, there is no question that when it does

This research was supported in part by Grant $\mathrm{MH}$ 30865 from the National Institute of Mental Health to Rutgers University, Irvin Rock principal investigator. The experiments described here were reported at the meeting of the Eastern Psychological Association, Philadelphia, Pennsylvania, April 1979.

Requests for reprints should be sent to Irvin Rock, Institute for Cognitive Studies, Rutgers-The State University, Newark, New Jersey 07102. change concomitantly with length, the kinetic depth effect is stronger and more readily and universally achieved.

The question we would like to pose is why does this transforming stimulus pattern generally produce an impression of a three-dimensional object? One answer might be that the combined length and orientation change constitutes the stimulus for depth, that is, directly leads to that percept much as a particular frequency of vibration of a sound wave reaching the ear constitutes the stimulus for the perception of a tone of a particular pitch. This kind of answer has in recent years been associated with the theory propounded by Gibson $(1950,1966,1979)$. Since, however, Gibson generally focused on the stimulus transformations of the entire optic array consequent on motion of the observer in a natural environment, an analysis of the kinetic depth effect in the laboratory would probably not be considered crucial or even relevant to his general theory. Even though one might read Wallach and O'Connell (1953) as expressing such a direct theory of their effect, it is best not to be too concerned here with whose theory we are investigating but rather to consider this interpretation as a logically possible one, whoever might advocate it. However, it is important to point out that although Gibson 
might not have chosen the kinetic depth effect as the ideal paradigm to represent or test the theory of direct perception, it does not follow that this effect is little more than an artificial laboratory phenomenon. On the contrary, if we think of object perception rather than of perception of extended planes, then the conditions isolated in the laboratory analysis of kinetic depth are precisely those that occur all the time in daily life when there is a change in the angular direction of any three-dimensional object, as a result of its movement or of the observer's movement.

The answer against which we would like to pit the direct theory is that the impression of depth results from a process like inference or problem solving. Such a view is generally associated with Helmholtz (1866) and others since who have followed his lead. However, the Helmholtzian view is that the inference process is necessarily built on induction from prior experience, whereas we would argue that an inference theory is defensible even without basing it on knowledge derived from prior experience. Moreover, this kind of theory has never been developed sufficiently to spell out what the inference process might be like or to cope with the specific problems that arise in the perception of particular objects and events. Therefore, in what follows, we will try to suggest a modified and somewhat more elaborated problem-solving theory that would be applicable to the problem we posed about the kinetic depth effect.

The broad outlines of such a theory would run somewhat as follows: The transforming stimulus poses the problem for the perceptual system as to what event in the world might be producing it. Hypotheses are generated that could do justice to that stimulus; that is, if such and such an event were occurring, it would produce just that stimulus. In the present case, two such hypotheses will be considered that would have that capability: (a) There is a line in a frontal plane that is simultaneously changing its length and orientation. This is a literal solution in that the percept corresponds directly with the stimulus transformations. One might speculate, therefore, that no prior experience is necessary for this hypothesis to be generated and also that, in terms of sequence, it is the first hypothesis to be generated. (b) There is a line of constant length rotating in depth about a particular axis. This is clearly not a literal solution but a constructive one. It might well arise by virtue of prior experience from daily life in which such transforming images were produced by rotating rigid objects. Alternatively, it is logically possible that the depth hypothesis is available on the basis of evolutionary "experience" rather than on the ontogenetic experience of the individual. In any event, this constructive solution might occur only after the literal solution has been entertained.

The first stage of the process, then, is the elicitation of one or more object or event hypotheses that would explain the stimulus transformation. Either certain features of the stimulus evoke a hypothesis, presumably on the basis of similarity to certain features of the solution, or it is elicited on the basis of a set or suggestion external to the stimulus as such. The second stage of processing consists of comparing stimulus and solution in terms of the adequacy of the match. Solutions will be rejected if they fail to account adequately for the stimulus transformation under discussion. They may also be rejected if while accounting for what is in the stimulus transformation, they require certain additional features to be present that in fact are not. For example, consider a drawing that consists of no more than a horizontal line across a page near the top. It might well suggest a horizon line dividing a ground plane from the sky, but a ground plane would presumably yield certain other stimulus features, and these are simply not present in the drawing. Their absence then constitutes negative evidence against that hypothesis. For a perceptual solution to be sustained, those stimulus components that ordinarily would be present, given such an object or event in the world, must be present to support that solution. One might think of or imagine a ground plane as present, but to perceive it requires support from the stimulus. However, the two hypotheses considered above for the case of the transforming stimulus both are acceptable in terms of these criteria of a stimulus-solution match.

Of these two possible solutions, the depth solution is preferred, since, given a sufficient 
period of observation, most observers arrive at it and, once having done so, do not, indeed generally cannot, avoid it and revert back to the literal solution. Thus, a fundamental problem here is to explain the preference for a certain perception given the ambiguity of the stimulus. We will therefore return to this question of preference. In any event, ambiguity is thus here explicitly acknowledged as an important characteristic of the stimulus. Conversely, it would seem that ambiguity must be denied by a direct perception theory for two reasons: first, because it can hardly be claimed that for every percept there is a unique stimulus and vice versa if, in fact, given that stimulus, the percept need not occur and second, because if ambiguity is acknowledged, then some mechanism or basis of selection must be introduced to explain the preferred outcome, and the necessity of doing so clearly undermines the essence and elegance of a direct perception theory.

Logically there can be no denying that the simultaneous length and orientation change of a line stimulus is ambiguous in that it can be produced in several different ways, and in fact Wallach and O'Connell (1953) did produce it either by an object rotating in depth or by a stimulus actually changing length and orientation. In the experiments reported here, the transforming image is never produced by an object rotating in depth. But beyond the issue of logical ambiguity is the matter of de facto ambiguity. The transforming stimulus does not always lead to the depth percept and, even when it does, is often preceded by a period when it is not so perceived.

In the experiments to be described below, certain changes in the typical kinetic depth display are deliberately introduced to investigate whether the rotation-in-depth outcome will still be preferred. We are predicting that in certain of these cases it will not because that perceptual outcome no longer constitutes the best or preferred solution to the problem. However, an advocate of the direct-perception theory might simply say that with any change in the stimulus, the prediction will now necessarily be different. In our opinion, that general answer is not an adequate defense. Clearly, there can be stim- ulus changes of all sorts that would not plausibly lead to a prediction of a change in perception. Indeed, in certain of our control conditions, the changes introduced do not lead to the abolition of the kinetic depth effect. Rather, it would have to be argued that the changes either eliminate the essential features of the stimulus -in this case the simultaneous length and orientation change -or add features that make the stimulus as a whole favor a different perceptual outcome. One might say that complex relationships among display elements are the hallmark of a sophisticated higher order direct perception theory. As to eliminating essential features, we do not introduce changes of this kind. The essential lengthorientation change will always be present. As to adding features, it remains to be discussed what can reasonably be claimed by a direct perception theory in each specific case.

In the experiments to be described, the transforming image is a luminous line simultaneously changing its length and orientation. The line, in fact, is oscillating in a frontal plane, thus changing its orientation. But, it is rotating behind and viewed through a rectangular aperture, thus also changing its length. The transforming image, therefore, is roughly the same as the one that would be produced by a thin rod rotating in depth about a vertical axis at its midpoint. ${ }^{1}$ Consequently, it was expected that when only the line was visible, the typical kinetic depth effect would occur.

However, the question of alternative solutions raised here was investigated by permitting the aperture to be visible. It was possible to cause it to appear either as an aperture or as a figural region. Thus, solutions other than the kinetic depth effect be-

\footnotetext{
' A rectangular aperture with straight horizontal upper and lower contours rather than curved contours generates the same transforming image of an oblique line rotating at its midpoint about a vertical axis only for a display at optical infinity. There are also certain other stimulus differences between this simulation method and the actual rotating display, such as the velocity of motion across the retina of the end points of the visible line. However, the equivalence is sufficiently close to disregard these differences as is empirically substantiated by the finding of kinetic depth perception in all of our conditions in which it was predicted.
} 


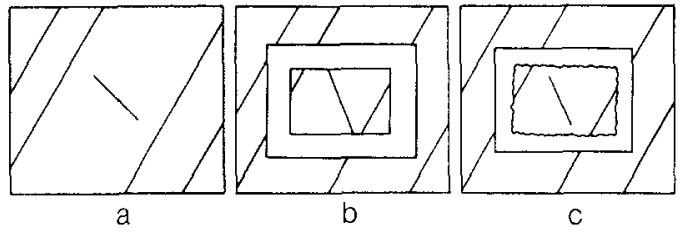

Figure 1. Displays used in Experiment 1: (a) Condition 1, (b) Condition 2, and (c) Condition 3.

came possible for each of these conditions, namely, either interpreting the line as oscillating in a frontal plane behind an aperture or as oscillating on the surface of a rectangle, the path of its ends being coterminous with the upper and lower contours of the rectangular figure. In other words, to consider the first of these, if the ends of the line always remain contiguous with the inner contour of a visible aperture, one interpretation of what is occurring might be that of a line oscillating behind the aperture in a frontal plane. This interpretation may then be preferred over that of a line rotating in depth, although the latter certainly remains a logically possible interpretation.

\section{Experiment 1}

\section{Method}

Subjects. A total of 30 subjects were employed, 10 in each of three separate conditions. They were undergraduate volunteers of both sexes, naive with respect to the phenomenon under study.

Procedure. Subjects were seated in a dark room 5 ft. $(1.5 \mathrm{~m})$ from a luminous line that oscillated through $90^{\circ}$ in a frontal plane from $45^{\circ}$ clockwise to $45^{\circ}$ counterclockwise. This was accomplished by placing a $1 / 8$ in. wide $\times 81 / 4$ in. $(.31 \mathrm{~cm} \times 20.96 \mathrm{~cm})$ long strip of luminous material on a disc that alternately changed its direction of movement at a speed of $8 \mathrm{sec}$ per cycle. The line was viewed under three conditions. In Condition 1 , the line could be seen through a $33 / 4$ in. $\times 5$ in. $(9.53$ $\mathrm{cm} \times 12.7 \mathrm{~cm}$ ) rectangular aperture, but the aperture itself was not visible because it was not made of luminous material. Consequently, the retinal projection of the line concurrently changed length and orientation, thereby fulfilling the essential stimulus requirement for the occurence of the kinetic depth effect (Figure la). In Condition 2 , the only variation was that the rectangle surrounding the aperture was luminous and, therefore, visible (Figure 1b).

In Condition 3, the aperture was not exposed, but a substitute aperture was visible. It was a luminous, irregularly contoured frame, similar to that seen in Condition 2. However, the upper and lower contours of this frame were not coterminous with the path of the ends of the line (Figure 1c). This condition was included as a control for the possibility that the presence of a visible aperture in Condition 2 might lead to a strong tendency to localize the line in the same frontal plane, via the equidistance tendency (Gogel, 1965), and thus oppose a depth outcome. The fact that there is a space between the ends of the line and the inner contours of the aperture rules out the interpretation that the line is rotating behind the aperture in a frontal plane. The contours of the aperture were made irregular to avoid the coincidence of the paths of the ends of the line being parallel to the visible contours.

For all conditions, the display contained a series of parallel, thin, obliquely oriented luminous lines that were included to enhance the perception of an aperture in Conditions 2 and 3. As shown in Figures $1 \mathrm{~b}$ and $1 \mathrm{c}$, portions of these lines were visible inside the aperture, thus supporting an impression of the inner region as ground rather than figure. In any of its positions, the oscillating line never contacted these oblique lines. The subjects viewed the display monocularly through a $1 / 2$ in. $(1.27 \mathrm{~cm})$ opening in a reduction screen, with their heads stabilized by a chin rest. Figure 2 illustrates schematically the arrangement of oscillating line, aperture, and luminous frame used in Condition 2. With appropriate changes (e.g., removing entirely or substituting a different luminous frame) this figure illustrates the other conditions. The oblique lines and visible aperture were mounted on a glass panel to which the actual aperture was affixed from behind. The glass panel was $1 / 4$ in. $(.64 \mathrm{~cm})$ in front of the oscillating line.

To avoid set effects, each subject was exposed to only one of the three conditions. The subjects were first asked to look at the stationary display and describe what they saw. Following this, they were told that the small luminous line would be set into motion. They were instructed to watch the path of motion carefully because later they would be given a thin wire rod with which to duplicate the perceived motion of this line. Thus, in the test, the subjects could either oscillate the rod in a frontal plane or rotate it in depth. It was believed that this task eliminated all ambiguities in verbal descriptions of what had been perceived. Each subject, in all conditions, viewed the oscillating line for $60 \mathrm{sec}$.

\section{Results}

Prior to the onset of motion, subjects perceived the luminous display of Condition 2 as a line behind a rectangular frame and the display of Condition 3 as a line inside an irregular frame. As to the main result concerning movement, in Condition 1, in which the transforming line alone was visible, 9 out of 10 of the subjects perceived the line rotating in depth; the other subject perceived the line oscillating in a frontal plane. The typical stimulus requirements for the occurrence of the kinetic depth effect prevailed, so the depth solution is to be expected here. Since the line in fact oscillated in a frontal plane, the perception of depth is illusory and 


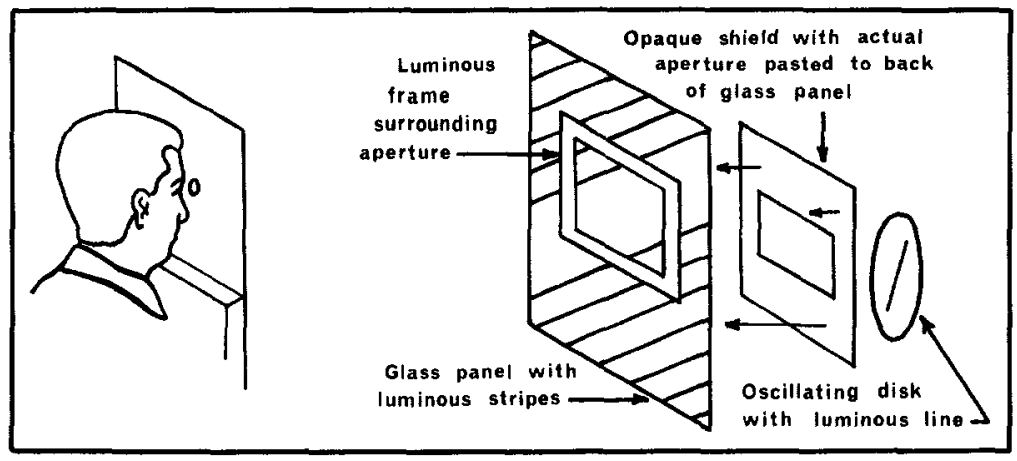

Figure 2. Schematic of display used in Experiment 1, Condition 2.

cannot be based on information picked up concerning the actual spatial arrangement.

In Condition 2, in which the aperture was visible, only 1 out of the observers perceived rotation in depth. The other 9 veridically perceived the objective state of affairs, a line of constant length oscillating in a frontal plane undergoing partial occlusion by the aperture. In Condition 3 , when the line was surrounded by - but the ends were not contiguous with - a visible aperture, 8 out of 10 of the subjects achieved a depth effect; the other 2 perceived the line oscillating in a frontal plane. This last finding appears to rule out an interpretation of the results of Condition 2 simply in terms of a tendency to perceive line and frame in Condition 2 as in one plane. There is clearly a significant difference between either Condition 1 and 3 on the one hand and Condition 2 on the other, $t(18)=6.0, \quad p<.01$, and $t$ $(18)=4.4, p<.01$, respectively. Instead, it seems that the crucial factor that determines whether or not a depth effect occurs is whether or not the path of the ends of the line is or is not coterminous with the visible inner frame contours.

\section{Experiment 2}

One might argue that the elimination of the kinetic depth effect in Condition 2 is not surprising, at least intuitively. The alternative possibility of perceiving a line of constant length undergoing partial occlusion is readily at hand. An advocate of a direct perception theory might say that we have simply introduced new stimulus conditions-those for a different kind of depth, namely, of an object in the frontal plane, but amodally behind an object in a different frontal plane, with the alleged stimulus conditions simply being "occlusion" and "disocclusion" (Gibson, 1979; Kaplan, 1969). If so, this condition can be thought of as representing conflicting determinants, with those favoring the kinetic depth effect losing out to those favoring the occlusion solution.

This brings us to Experiment 2. Here, the line is made to appear on a rectangular surface, not behind a rectangular aperture. Under these conditions it can no longer be argued that the kinetic depth interpretation is simply displaced by one that is more appropriate to the total stimulus context, namely that of occlusion/disocclusion. For now that interpretation is not likely to occur, whether because, as we would say, the figure-ground organization will no longer support it or for whatever reason an advocate of the direct perception theory might wish to advance. If that interpretation is not likely to occur, then the elimination of the kinetic depth interpretation cannot be explained in terms of a different direct perception prediction as seems to be possible in the case of Experiment 1.

\section{Method}

Subjects. A total of 20 subjects were employed, 10 in each of two separate conditions. They were undergraduate volunteers of both sexes, naive concerning the phenomenon under study.

Procedure. There was one main difference between this and Experiment 1. In Experiment 1, a display was presented that would appear as an aperture behind 


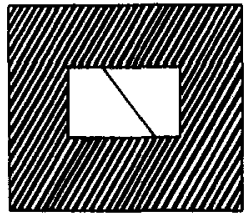

a

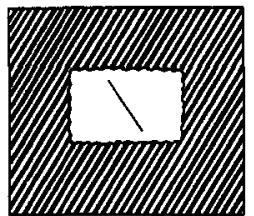

b
Figure 3. Displays used in Experiment 2: (a) Condition 1 and (b) Condition 2.

which the line was visible. In Experiment 2, a display was created with thicker and more closely arranged oblique lines outside a rectangular contour, and the oblique lines were no longer visible inside as was the case in Experiment 1. Thus, as the reader can see in Figure $3 \mathrm{a}$, the conditions now favored perception of a solid rectangularly shaped figural region instead of an aperture region. Consequently, it was assumed that the luminous line would appear to be on this rectangular surface. Thus, the alternative solution available in Experiment 1 -a line of constant length undergoing partial occlusion by an aperture - would be unavailable because the line would be seen on a surface, not through an aperture.

There were two conditions analogous to those in Experiment 1 . In Condition 1 , the line was expected to be seen on the surface of a rectangle whose upper and lower contours were coterminous with the path of the ends of the line (Figure 3a). In Condition 2, the path of the ends of the line was not coterminous with the contours of an irregularly shaped rectangular figure (Figure $3 b$ ). These oblique line displays were pasted on the glass panel instead of the displays used in Experiment 1 (see Figure 2). In all other respects the apparatus and procedure were the same as in Experiment 1.

\section{Results}

In both conditions, before motion was introduced, subjects reported perceiving a luminous line lying in front of, or directly on, a solid figural region. It was important to establish this fact because objectively the rectangular region was in fact an opening. The main results concerning movement can be simply stated. In Condition 1, all 10 of the subjects perceived oscillation in a frontal plane rather than rotation in depth. No subject had any impression of occlusion/disocclusion. The line seemed to change its length as it rotated on the rectangular surface. In Condition 2, only 3 out of 10 of the observers had this kind of perception. The remaining 7 perceived motion in depth. The difference is significant, $t(18)=4.6, p<.01$.

\section{Experiment 3}

In the final experiment, we investigated whether the effect obtained in the first two experiments would still occur if illusory contours of the visible aperture or figural region were substituted for real contours. If so, it would imply that what matters is how the rectangle display is perceived rather than the presence of specific stimulus components. It would suggest that whether or not the oscillating line is perceived in depth depends on the perception of the static display.

\section{Method}

Subjects. A total of 30 subjects were employed, 10 in each of three separate conditions. They were undergraduate volunteers of both sexes, naive with respect to the phenomenon under study.

Procedure. There were no differences in instruction or procedure, except that illusory contours were used to create stimulus conditions analogous to those of Experiments 1 and 2.

In Condition 1, the intention was to simulate an aperture (as in Experiment 1) by the use of illusory contours. To this end, four luminous circles were sectioned and the parts arranged to create the impression of an illusory frame. Thin oblique lines were again used to encourage the perception of an aperture (Figure 4a.). In Condition 2, illusory contours were used to simulate a rectangular opaque surface (as in Experiment 2). To this end, four partial circles were used, which, in conjunction with the elimination of the oblique lines in the central region, were expected to lead to the illusory perception of a solid figural region (Figure $4 \mathrm{~b}$ ). The third condition was constructed in the same way as the first; however, the sectioned circles were placed so that the path of the ends of the oscillating line was not coterminous with the illusory inner contours of the frame. This condition was included to control for the possibility that the luminous lines were casting enough light to reveal the actual contour of the presumably invisible aperture occluding the moving line in Condition 1 . Condition 3, therefore, would be decisive as to whether or not the illusory contours were really illusory. Assuming they were illusory, this condition also serves as a control analogous to that of Condition 3 of Experiment 1 (Figure 1c). The kinetic depth effect should be expected to occur.

\section{Results}

The results were analogous to the previous experiments. In Condition 1, all subjects per-

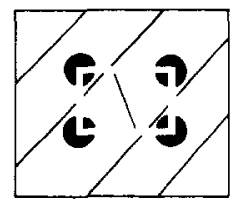

a

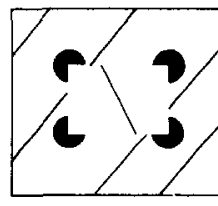

b

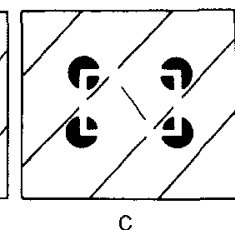

Figure 4. Displays used in Experiment 3: (a) Condition 1, (b) Condition 2, and (c) Condition 3. 
ceived an illusory aperture behind which 8 out of 10 of the subjects perceived a line oscillating in a frontal plane undergoing partial occlusion. The other 2 perceived rotation in depth. In Condition 2, all subjects described the display as containing a solid rectangular region, and 7 out of 10 perceived a line oscillating in a frontal plane; the other 3 perceived motion in depth. Condition 3 was described by the subjects in the same way as Condition 1, namely, as a hollow rectangular perimeter, and 8 out of 10 of the subjects achieved a strong depth effect; the other 2 perceived a line oscillating in a frontal plane. If the real aperture had been visible, the results for Condition 3 should have been the same as Conditions 1 and 2, but they were opposite. Taking Condition 3 as a control, both Conditions 1 and 2 differ significantly from it, $t(18)=3.4, p<.01$, and $t(18)=2.6, p<.01$, respectively. Therefore, this interpretation can be ruled out; instead, it seems that the outcome of Experiments 1 and 2 can be achieved even when actual contours are not physically or retinally present.

\section{Discussion}

As noted earlier, it might be argued that the outcome of Experiment 1, in which the line appeared to be behind an aperture, represents no challenge to a direct theory of perception. Presumably, the stimulus conditions introduced favor the perception of a line undergoing occlusion and disocclusion, thus explaining why the rotary depth percept does not arise. But on further reflection, it would seem that a direct theory of perceived occlusion and disocclusion also runs into difficulties.

It would take us too far afield to spell this out in detail, but the difficulty reduces to the fact that a region undergoing accretion or deletion will only appear to be behind an adjacent region if the latter is organized as figure (Rock \& Gilchrist, 1975). After all, the length of the visible line increases and decreases in all conditions of all of the experiments reported here, but the occlusion-disocclusion outcome is really only plausible when the line terminates in a region that is figural (Condition 2, Experiment
1 and Condition 1, Experiment 3). Thus, to invoke a stimulus explanation here is to commit the experience error (Köhler, 1947), since what is figure and what is ground is not simply given in the stimulus.

But even if there were no difficulties with a direct theory of occlusion-disocclusion, there is the further matter of explaining why the changing stimulus in Experiment 1 should now be expected to lead to that perceptual outcome. Must we say that the stimulus is no longer one that should be expected to lead to the kinetic depth outcome? If not, why not? Most of the essential ingredients are still present. Or must we rather say that a conflict now exists, in that the stimulus is appropriate for either of two perceptual outcomes? In that case, how was the preference for occlusion-disocclusion predicted? Of course, similar problems exist for the problem-solving view. How would we explain the preference? We will return to a discussion of this problem shortly. In any event, in Experiment 2 , the line is made to appear on a rectangular surface, not behind a rectangular aperture. Here the alternative of a line undergoing occlusion and disocclusion simply does not occur. Thus, if in fact the kinetic depth effect is here largely abolished, the question arises as to why, from the standpoint of a direct perception theory, that should be predicted.

Can a direct perception theory deal with the phenomenon of illusory contour? If so, one might argue that Experiment 3 introduces no difficulties for such a theory beyond those that may or may not exist for it in the two previous experiments. This is not the place for a full discussion of this phenomenon, but we would argue that it can hardly be done justice simply by referring to the stimulus characteristics of the kind of pattern that typically leads to such illusory perception. Thus, just to hint at some of the difficulties such a theory would encounter, there is the fact that with the appropriate displays, the illusory contours may or may not be perceived, that to perceive them implies a figure-ground reversal, and finally that one condition leading to this perception is incompletion of stimulus fragments when what is incomplete depends on familiarity with the complete object and thus cannot be 
defined simply in terms of the stimulus (see Rock \& Anson, 1979).

Even if it were possible to deal adequately with illusory contours in terms of a direct theory of perception, however, this would still fail to come to grips with the link between perceiving figures with such contours and the perception of the oscillating line in Experiment 3. We take the position that the perception of the aperture or the opaque figure occurs first, indeed before the line is set in motion, and this is true for Experiments 1 and 2 as well. It is that perception which then sets the stage for how the oscillating line will be perceived. But even if the perception of the static figure is not temporally prior to the perception of the line's motion, the perception of the latter is logically dependent on the perception of the former. If that illusory contour perception does not occur-and it would not be difficult to arrange conditions in which it would not-then there would be little reason to expect any outcome other than the kinetic depth solution in all conditions. Theoretically, what is important here is that there are occasions, of which this is one, when one perception is a function of or is logically dependent on another perception. This being the case, it is evident that the final perception is not direct, if direct is used in the sense defined in this article.

We must now consider in more detail how we would explain the various preferences encountered here in terms of a problem-solving theory. Specifically, we first must try to account for the preference for the kinetic depth solution under the typical laboratory conditions such as those in Experiment 1, Condition 1, and in those conditions in which we predicted it, namely, Experiment 1, Condition 3; Experiment 2, Condition 2; and Experiment 3, Condition 3. Although none of these last three cases are pure kinetic depth conditions, in that in each of them are certain additional stimulus components, they are not components that should be expected to matter. Thus, in passing, we wish to emphasize that a new context does not necessarily affect the outcome. In all of these cases, the fact is that the line that simultaneously changes its length and orientation is relatively isolated. Thus, although the transformation is ambiguous, the kinetic depth solution accounts for the covariation of length and orientation change elegantly and without the need to accept what would otherwise be a coincidental covariation. In other words, with the line rotating about a vertical axis, the line's retinal image would simultaneously change in both length and orientation, by virtue of changing perspective foreshortening, so that this covariation is accounted for by a single cause, so to speak. With the line oscillating in a plane, however, the change in length correlated with the change in orientation is not at all accounted for. It is inexplicable and coincidental.

Consider next the static display in Experiment 1 , Condition 2, in which a rectangular frame is seen surrounding a central region perceived as aperture or ground in which the transforming line is located. Given that perception of the central region in the static display, supported as it is by the oblique lines running across the field and through this region, the question to be addressed is why there is a preference to perceive the transforming line as one of constant length rotating in a frontal plane behind the frame. Logically it is possible to perceive the line as rotating in depth (the kinetic depth effect) either behind or in front of the frame. But either of these perceptions would imply that the path of the ends of the line in three-dimensional space precisely projected to the eye along the upper and lower inner contours of the aperture. That would be a highly coincidental or accidental state of affairs, and thus, we are arguing, the perceptual system rejects it.

This is how we would explain why the kinetic depth interpretation will not be preferred in this condition. That the perception of oscillation of a line in a frontal plane with partial occlusion of its ends is preferred over the perception of a line oscillating in a frontal plane and changing its length is also not hard to understand. Change of length would imply that the line was in the same plane as the frame rather than behind it. In the first place, even for the static display, the line would be expected to be-and in fact isperceived as extending behind the frame. We have here the conditions for interposition or completion. With the line oscillating, the 
conditions for interposition remain in force, but even if they were not, the occlusion-disocclusion solution should be preferred because the covarying change of line length and orientation is accounted for by the perception of an interposed opaque frame, whereas in the absence of that solution, the covariation remains unaccounted for.

This analysis makes all the more puzzling the outcome of Condition 1 of Experiment 2. For here the line is perceived as inexplicably changing length as it oscillates. Thus, the solution here accepts a coincidental state of affairs. Why the perceptual system does not reject this solution in favor of either the kinetic depth or occlusion-disocclusion solution requires an answer. The latter solution would entail a contradiction, namely, that the inner region cannot at one and the same time be perceived as both opaque (or figural) and as opening (or ground). Since the conditions were deliberately made effective for the perception of the inner region as figure, the line cannot then be seen as going behind it-unless, we might add, a figure-ground reversal were to occur. But why then is the kinetic depth solution not preferred? We have already explained in the case of Experiment 1 how that would entail acceptance of the coincidence of the end points of the line remaining coterminous with the contours of the central region. That same reasoning applies to Experiment 2. Thus, it would seem to come down to a contest between two kinds of coincidence. Either the line is seen to oscillate in a frontal plane coincidentally covarying in length and orientation, or it is seen as rotating in depth in front of the central figural region with its retinal projection coincidentally terminating along the projection of the contours of the figural region. Apparently, the latter coincidence is taken as more unlikely than the former, and thus the kinetic depth solution is rejected. We cannot at this time account for this ranking by the perceptual system of different coincidences in terms of any formal principle.

As can be seen from this discussion, various meanings of coincidence can be distinguished. The two most relevant to these experiments concern either unexplained

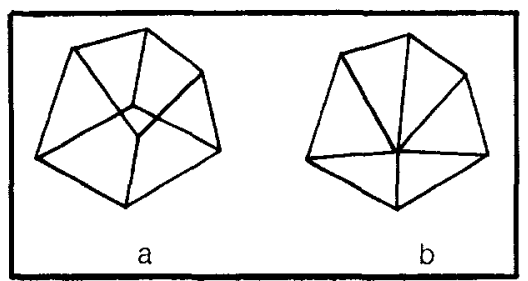

Figure 5. Drawings of irregular "wire" figures (after Kanizsa, 1975).

covariation-as in the case of length and orientation change in the typical kinetic depth display - or certain regularities within the proximal stimulus that would occur only by virtue of unique, accidental, or special spatial arrangements of observer vis-à-vis objects-as in the special displays used in these experiments. Another example of this latter meaning would be a drawing of an irregular wire cube that yields a retinal image such as that shown in Figure 5b, which will occur only if the far and near corners of the cube are both along the line of sight to one eye. For all other positions the retinal image would be like that shown in Figure $5 \mathrm{a}$. Given the coincidental nature of such a projection ( $5 \mathrm{~b})$, the perceptual system favors a solution that does not entail it, in this case that the object is two dimensional, whereas the drawing in 5 a will typically be perceived as three dimensional.

However this is not the place for an extended treatment of this hypothesis. ${ }^{2}$ The notion of coincidence-rejection based on unique spatial arrangement is similar to the concept of general viewpoint that has emerged independently among investigators in the field of artificial intelligence (Huffman, 1971). This states that we tacitly assume that the appearance of some picture feature will not change qualitatively if one changes one's viewpoint slightly. Thus, the nature of the momentary percept is held not to be simply an accident of viewpoint.

There are, of course, other views about preference in ambiguous stimulus conditions. Chief among these is the notion of preference based on prägnanz, or simplicity,

${ }^{2}$ For further discussion of the notion of coincidence as a basis of preference, see Rock (1975), Rock and Gilchrist (1975), Sigman and Rock (1974), and Rock and Anson (1979). 
first propounded by Gestalt psychology (Koffka, 1935; Kopfermann, 1930) and later by those influenced by information theory (Attneave, 1954; Hochberg \& Brooks, 1960; Hochberg \& McAlister, 1953; Restle, 1979). In these later versions, the preference is always for the perceptual outcome that is most economically encoded. Clearly, to avoid circularity here, it is necessary to have criteria that enable one to predict what is or is not "simple" in any given situation. Such criteria have been proposed for several perceptual phenomena such as pictorially produced depth, motion of dots, and the like, but as yet none have been advanced that would be directly applicable to the kinetic depth effect.

We can, however, hazard a guess as to what form such a theory might take in connection with this effect. It might be argued that it is simpler-more economical-to describe or encode a stimulus line covarying in length and orientation as a rigid object rotating in the third dimension about an axis than to describe it as simultaneously changing length and orientation in a frontal plane. That may well be true, but the claim is difficult to assess in the absence of a more specific theory. It might be relevant to point out, however, that any perception that entails the third dimension of space is at least in one sense less simple than one that does not.

How would this approach to preference fare in dealing with the present experimental results? It is difficult to see why the economy presumably gained by the kinetic depth interpretation would now be lost simply because of the presence of the additional structures used in Experiments 1, 2, and 3. That it is not simply the presence of such structures that matters is shown by the results of the several control conditions in which the kinetic depth solution is preferred. These control experiment results also show that it is not merely a matter of economy gained by interpreting all components as in one plane, since the outcomes here are the opposite. Thus, it would seem that a simplicity theory will have to come to grips with the features of these experiments that we have concluded are crucial, namely, the coterminous state of affairs that exists between the ends of the visible line and the contours of the inner region of the display. It is thus premature to judge the capability of such a theory to deal with the effects described here, but in the meanwhile we have predicted these effects on the basis of an alternative theory. It might be worth noting that these two approaches to the problem of preference are not necessarily mutually exclusive, at least with respect to differing perceptual paradigms or domains. However, it is clearly desirable to be able to pit them against one another when it comes to any specific perceptual effect.

It is important to be clear that the simplicity theory stresses economy of perceptual processing, whereas the coincidence theory suggested here stresses probability of veridical outcome as part of a process of problem solving. "Simplicity of outcome" on the one hand and "elegance," "commonality of cause," or "avoidance of coincidence" on the other, are perhaps similar concepts in some respects, but the former entails selection from among the set of possible perceptual descriptions, whereas the latter refer to characteristics of the solution process, namely, selection on the basis of relating the stimulus to alternative hypothetical external objects or events. Thus, a percept that is interpretable without accepting coincidence might be one that would require rather complex description. In other words a noncoincidental explanation or solution is not the same thing as a simple or economically describable percept.

The findings reported here are analogous to certain recent experiments on other perceptual phenomena in which it was also shown that the stimulus conditions believed to be necessary and sufficient to produce a particular perception will not do so when other solutions are preferred. For example, it was shown that alternating stimulation of spatially separate retinal loci at the appropriate rate will not yield a stroboscopic motion effect if the sudden appearance and disappearance of the stimulus objects (dots) can be interpreted otherwise, namely, as objects undergoing occlusion and disocclusion by a moving opaque surface (Sigman \& Rock, 1974). We would maintain that ordinarily the stroboscopic effect is the best solution to the problem posed by the stimulus 
sequence, since the sudden appearance and disappearance of the objects is otherwise inexplicable. But, when introduced, the presence of a visible opaque surface moving back and forth supports an occlusion-disocclusion interpretation, and this interpretation avoids what would otherwise be a spatial and temporal coincidence if the dots were to be seen as simultaneously moving in antiphase to that of the moving surface.

We interpret these experiments as evidence against any theory which maintains that perception is based directly and exclusively on either an absolute or a higher order attribute of the proximal stimulus. Rather, the experiments support Helmholtz's (1866) view that the stimulus is interpreted in terms of what it most probably represents in the world.

\section{References}

Attneave, F. Some informational aspects of visual perception. Psychological Review, 1954, 61, 183-193.

Gibson, J. J. The perception of the visual world. Boston: Houghton-Mifflin, 1950.

Gibson, J. J. The senses considered as perceptual systems. Boston: Houghton-Mifflin, 1966.

Gibson, J. J. The ecological approach to visual perception. Boston: Houghton-Mifflin, 1979.

Gogel, W. Equidistance tendency and its consequences. Psychological Bulletin, 1965, 64, 153-163.

Helmholtz, H. von. Handbuch der physiologischen Optik. (Vol. 3). Leipzig, Germany: Voss, 1866.

Hochberg, J., \& Brooks, V. The psychophysics of form:
Reversible perspective drawings of spatial objects. American Journal of Psychology, 1960, 73, 337-354.

Hochberg, J., \& McAlister, E. A. A quantitative approach to figural "goodness." Journal of Experimental Psychology, 1953, 46, 361-364.

Huffman, D. A. Impossible objects as nonsense sentences. In B. Meltzer \& D. Mickie (Eds.), Inactive intelligence 6. Edinburgh, Scotland: Edinburgh University Press, 1971.

Johansson, G., \& Jansson, G. Perceived rotary motion from changes in a straight line. Perception \& Psychophysics, 1968, 4, 165-170.

Kanizsa, G. The role of regularity in perceptual organization. In G. B. Flores d'Arcais (Ed.), Studies on perception. Milan, Italy: Martello, 1975.

Kaplan, G. A. Kinetic disruption of optical texture: The perception of depth at an edge. Perception \& Psychophysics, 1969, 6, 193-198.

Koffka, K.Principles of Gestalt psychology. New York: Harcourt, Brace, 1935.

Köhler, W. Gestalt psychology. New York: Liveright, 1947.

Kopfermann, H. Psychologischen Untersuchungen uber die Wirkung zweidimensionäler Darstellungen körperlicher Gebilde. Psychologische Forschung, 1930, 13, 293-364.

Restle, F. Coding theory of the perception of motion configurations. Psychological Review, 1979, 86, 1-24.

Rock, I. An introduction to perception. New York: Macmillan, 1975.

Rock, I., \& Anson, R. Illusory contours as the solution to a problem. Perception, 1979, 8, 665-681.

Rock, I., \& Gilchrist, A. The conditions for the perception of covering and uncovering of a line. American Journal of Psychology, 1975, 88, 571-582.

Sigman, E., \& Rock; I. Stroboscopic movement based on perceptual intelligence. Perception, 1974, 3, 9-28.

Wallach, H., \& O'Connell, D. N. The kinetic depth effect. Journal of Experimental Psychology, 1953, $45,205-217$.

Received September 13, 1979 